九州大学学術情報リポジトリ

Kyushu University Institutional Repository

\title{
Oscillatory Behavior of Enzymatic Feedback System
}

Okamoto, Masahiro

Laboratory of Sericultural Chemistry, Faculty of Agriculture, Kyushu University

Aso, Yoichi

Laboratory of Sericultural Chemistry, Faculty of Agriculture, Kyushu University

Hayashi, Katsuya

Laboratory of Sericultural Chemistry, Faculty of Agriculture, Kyushu University

https://doi.org/10.5109/22893

出版情報：九州大学大学院農学研究院紀要. 20 (3/4)，pp. 105-116，1976-10. Kyushu University バージョン：

権利関係 : 


\title{
Oscillatory Behavior of Enzymatic Feedback System
}

\author{
Masahiro 0 kamoto, $\mathrm{Y}$ oichi Aso and Katsuya Hayashi \\ Laboratory of Sericultural Chemistry, Faculty of Agriculture, \\ Kyushu University, Fukuoka
}

(Received December 12, 1975)

\begin{abstract}
It has been well recognized that many enzymatic chain reactions and metabolisms in living organisms exhibit an oscillatory character with respect to the concentration of an intermediate. Some kinds of oscillation observable on the metabolic intermediate were presumed to be due to the feedback control system in the enzymatic chain reaction, which can produce the time-retardation function.

Analog computer simulation in the present study revealed an appropriateness of the above assumption. Since the feedback system has been recognized to be the universal mode for the regulation of enzymatic chain reactions in vivo, it was concluded that the oscillatory behaviors in enzymatic chain reactions may not be particular but general one.
\end{abstract}

\section{INTRODUCTION}

Many investigators have experimentally proved that oscillatory chemical reactions exist in the homeostatic regulation systems (Goodwin, 1965; Higgins et al., 1973; Sompayrac and Maal $\phi$ e, 1973). The various oscillatory behaviors observable in living organisms such as secretions of hormones, beat of heart, circadian rhythms or interacting population (Pavlidis, 1973) may probably be fundamentally caused by chemical events. Therefore, the quantitative explanation of the mechanism of chemical oscillation in vivo may provide some information for understanding the mechanisms by which the highly organized oscillations are generated.

An oscillatory phenomenon in physical or technological system, as seen in the behavior of a pendulum, is essentially due to the inertia term. A chemical system is characterized to have no inertia term. Chemical reactions can occur or stop at the moment, not producing time-retardation by the inertia term. Therefore, in a system having no inertia term, the periodic response would be caused by another factors which are able to act on a system in the same way as the physical inertia term.

On the other hand, it is well known that a typical oscillation with sine or cosine curve is easily generated by a looped (feedback) electronic circuit. A looped pathway is considered to be a retardation-realizing device, corresponding to the effect of the physical inertia term. From this fact, it is expected that the chemical oscillation would be derived from a chemical system involving a looped step or feedback system. Actually, Morales and McKay (1967) have first clarified the relationship between the feedback control system and the biochemical oscillation. 
Many reactions in living organisms are characterized to exhibit a nonlinear behavior. Feedback control systems in vivo could not simply be analyzed by the transfer function. The most suitable method for analyzing such the systems is thought to be one involving the numerical computation or the computer simulation of the mathematical model.

The present report deals mainly with the oscillatory characteristics of biochemical feedback system; the parameter-set in the feedback system which can realize the oscillation, changes in the stationary concentrations of the intermediates caused by an external perturbation and the conditions under which the concentration of the final intermediate in the feedback loop was maintained in constant value without suffering the effect of the perturbation, were specially investigated by the computer simulation.

\section{COMPUTATION}

The following reaction scheme was assumed to be a typical biochemical feedback system, where $X_{i}$ represents the reactant or intermediate, $Y_{1}$ and $Y_{2}$ are input and output of the system and both are assumed to be constant values during the reaction. $k_{1}, k_{2}$ and $k_{3}$ represent the reaction rate constants of

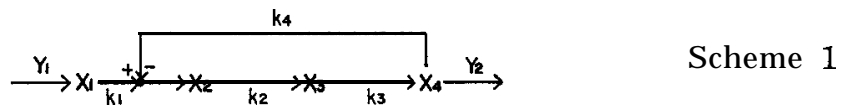

corresponding step. $k_{4}$ is considered to be a feedback constant controlling the weight of contribution of the concentration of $X_{4}$ to feedback summing point. At the summing point between $X_{1}$ and $X_{2}$, following operation is assumed:

$$
Z=\frac{k_{1}}{k_{4} \bar{X}_{4}}
$$

The rate equation of Scheme 1 in the form of simultaneous differential equation may be written as:

$$
\left\{\begin{array}{l}
\frac{d X_{1}}{d t}=Y_{1}-\left(k_{1} / k_{4} X_{4}\right) X_{1} \\
\frac{d X_{2}}{d t}=\left(k_{1} / k_{4} X_{4}\right) X_{1}-k_{2} X_{2} \\
\frac{d X_{3}}{d t}=k_{2} X_{2}-k_{3} X_{3} \\
\frac{d X_{4}}{d t}=k_{3} X_{3}-Y_{2}
\end{array}\right.
$$

The set-up diagram of the equation (2) for analog computer is shown in Fig. 1. Bold lines indicate the feedback path. The simulations were conducted with changing the loop size, values of the rate constants and the initial concentration of $X_{4}$. All computations were performed by a Hitachi analog computer Model ALS-220 in the Biotron Institute of Kyushu University. 


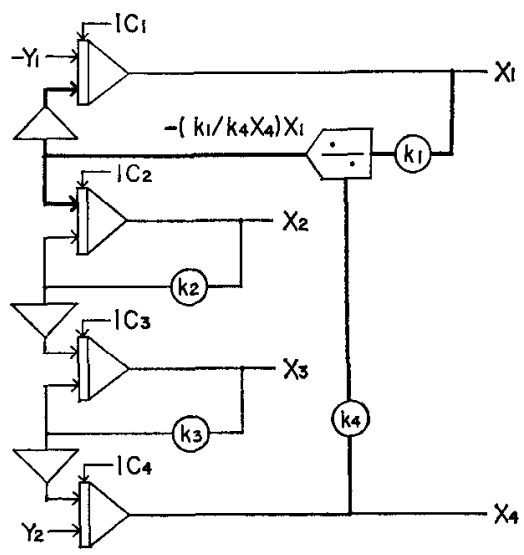

Fig. 1. Set-up diagram of equation (2) for analog computer.

\section{RESULTS AND DISCUSSION}

\section{Pattern of oscillation}

The typical patterns of concentration of $X_{i}$ vs time and phase diagram, which are sampled from various patterns obtained by the present simulation, are shown in Figs. 2-6 to classify roughly the oscillatory patterns.

Figure 2 shows a non-oscillatory pattern: (A) is the computed time-course of the reaction product in the feedback system, $X_{4}$, and (B) the phase diagram (trajectory) between two intermediates, for instance $X_{3}$ and $\mathrm{X}$,. The ordinate of (B) indicates the concentration of an intermediate and the abscissa the concentration of another intermediate. With the analysis of phase diagram, the transition behavior of the system can be predicted according to the shape of the trajectory. In Fig. $\mathrm{Z}(\mathrm{B})$, the trajectory converged to a certain point, the lower end of the curve.
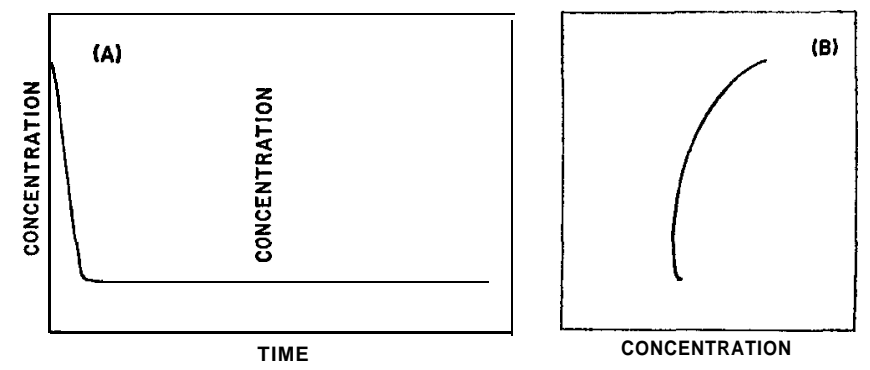

Fig. 2. Non-oscillatory pattern. (A) Time-course, (B) phase diagram.

Figure 3 shows a typical pattern of damped oscillation. The trajectory converges to a center point with drawing a swirl. The sustained oscillation is shown in Fig. 4. In this case, the average value of $X_{4}$ at the stationary state is lower than the value of initial $X_{4}$. The trajectory of this type draws a limit 

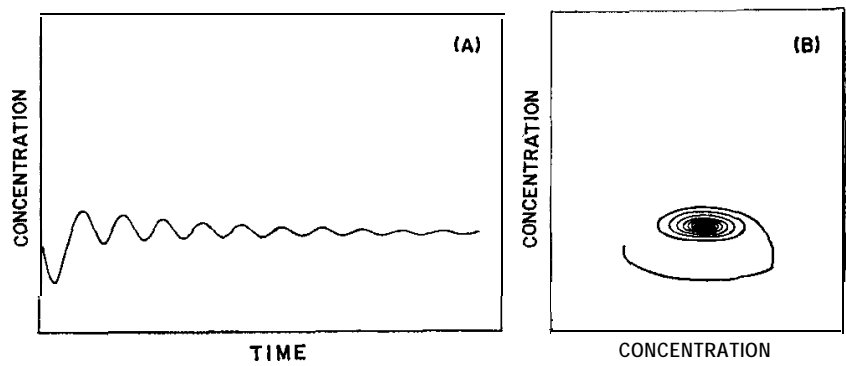

Fig. 3. Damped oscillation. (A) Time-course, (B) phase diagram. In (B), the trajectory converges to a center point.
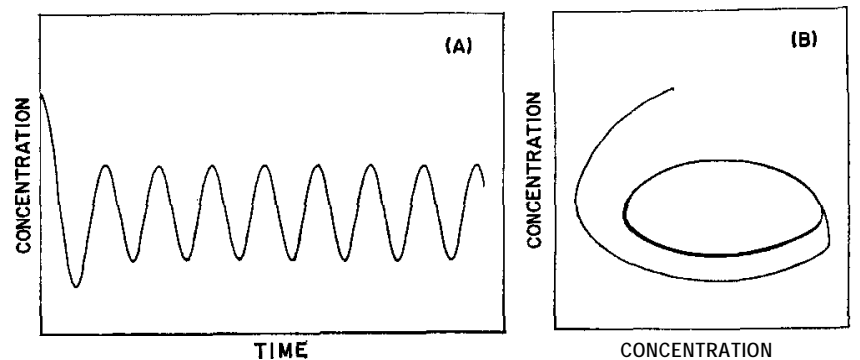

Fig. 4. Sustained oscillation. In phase diagram, starting point is at the upper center. This type of oscillation is called sustained inside.
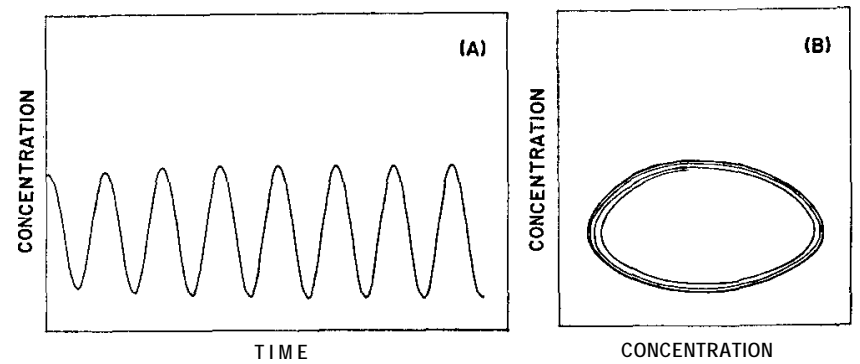

Fig. 5. Sustained oscillation. In phase diagram, starting point is in limited cycle. This type of oscillation is called sustained outside.
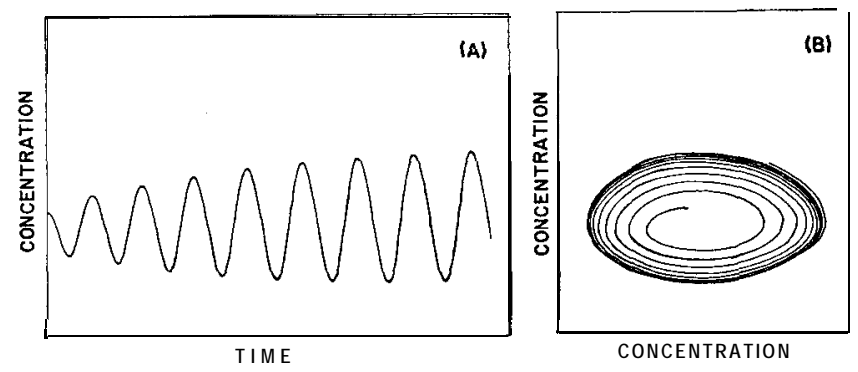

Fig. 6. Divergent oscillation. In phase diagram, starting point is inside of swirl. 
cycle. Figure 5 also shows a pattern of sustained oscillation. However, this holds a higher average value of $X_{4}$ at the stationary state than the initial value of $X_{4}$. The limit cycle in this case is at the outside of the starting point. Figure 6 shows the typical pattern of divergent oscillation. The trajectory diverges with drawing a swirl outward from the starting point. The oscillating patterns in Figs. 3-6 are respectively represented as Damped, Sustained inside, Sustained outside and Divergent.

\section{Position of summing point and oscillation}

It is assumed in general that an end-product of enzymatic chain reaction inhibits some enzyme in the same chain by negative feedback, keeping itself the stationary concentration at the desired constant level. In this simulation, therefore, the feedback system is assumed to be framed in the way that the end-product $X_{4}$ is fed back to a preceding reaction step. In the case of that

$$
\stackrel{r_{1}}{\longrightarrow} x_{1} \longrightarrow x_{2} \longrightarrow x_{3} \rightarrow \stackrel{1}{\longrightarrow} \underset{X_{4}}{\stackrel{Y_{2}}{\longrightarrow}} \quad \text { Scheme } 2
$$

the summing point of feedback was between $X_{3}$ and $X_{4}$ (Scheme 2), the timecourse of $X_{4}$ was entirely similar to that shown in Fig. 2(A), holding a nonoscillatory constant value at the stationary state. The typical pattern in the case of that $X_{4}$ inhibits the enzyme which catalyzes the reaction between $X_{2}$ and $X_{3}$ is shown in Fig. 7. The $X_{4}$ showed the damped oscillation.

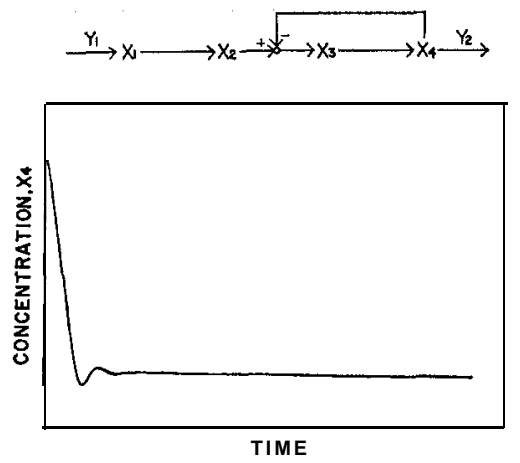

Fig. 7. Damped oscillation. The scheme drawn on the figure was analyzed.

In order to see the effect of another operation (equation (3)] at the summing point, a scheme shown on Fig. 8 was also assumed.

$$
Z=k_{1}-k_{3} X_{3}
$$

This system realizes only negative feedback because the non-negativity of $Z$ value, while in the equation (1) the region of $Z$ value is $Z \$ k_{1}$, indicating the possibility of realizing a negative or positive feedback. The computed results are shown in Fig. 8. The end-product of the system, $X_{3}$, shows also a kind of damped oscillation in the transition state. Thus, the difference in the operation mode at the summing point did not change the basic oscillatory behavior of the system, if only the negative feedback was considered. 

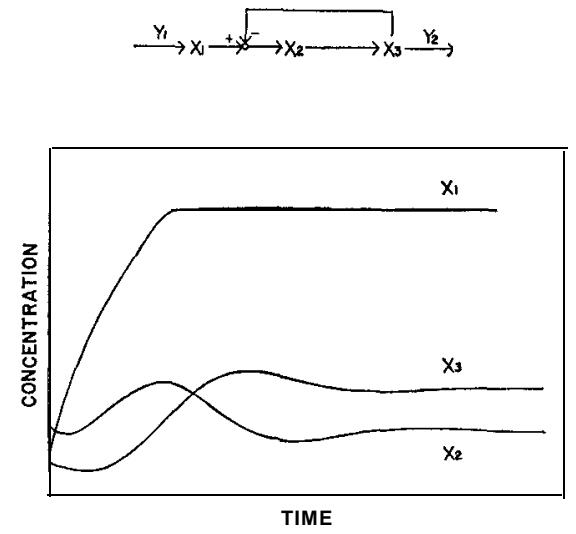

Fig. 8. Damped oscillation. The scheme drawn on the figure was analyzed with assuming the operation at the summing point is represented by equation (3).

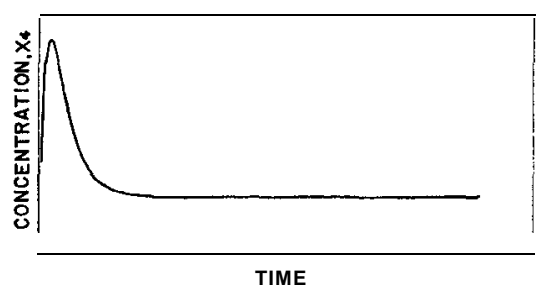

Fig. 9. Typical non-oscillatory pattern. Scheme 1 was analyzed.

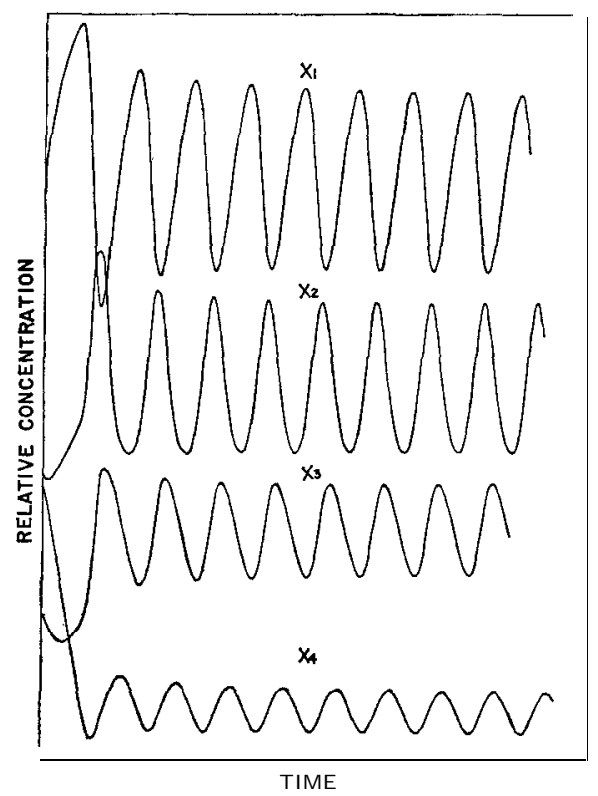

Fig. 10. Typical oscillatory pattern of Scheme 1 . 
The time-courses of $X_{i}$ in the system in which the summing point is between $X_{1}$ and $X_{2}$ showed various profiles according to the parameter-set used for the computation. Under a certain parameter-set, $X_{4}$ kept a constant value after overshot the initial value, as shown in Fig, 9. With another parameterset, the sustained oscillations of all $X_{i}$ were observed as shown in Fig. 10. Thus, it was found that for the appearance of an obvious oscillation there was a limitation of the loop size, when the positive feedback was not taken into the consideration. In the following computer simulations, only Scheme 1 was analyzed.

\section{$\boldsymbol{k}_{1} / \boldsymbol{k}_{\mathbf{4}}$ Ratio and oscillation}

The simulations of Scheme 1 were performed with various combinations of $k_{1}$ and $k_{4}$ under fixing the values of other parameters to clarify the effects of $k_{1}$ and $k_{4}$ on the oscillatory behavior of the system. The sustained oscillations were observed with the combinations listed in Table 1 . The detailed oscillatory profiles are shown in Fig. 11. From these results, it is obvious that for the appearance of the sustained oscillation, $k_{1} / k_{4}$ ratio should be limited to a narrow range, while the individual $k_{1}$ and $k_{4}$ are allowed to have a considerably extensive

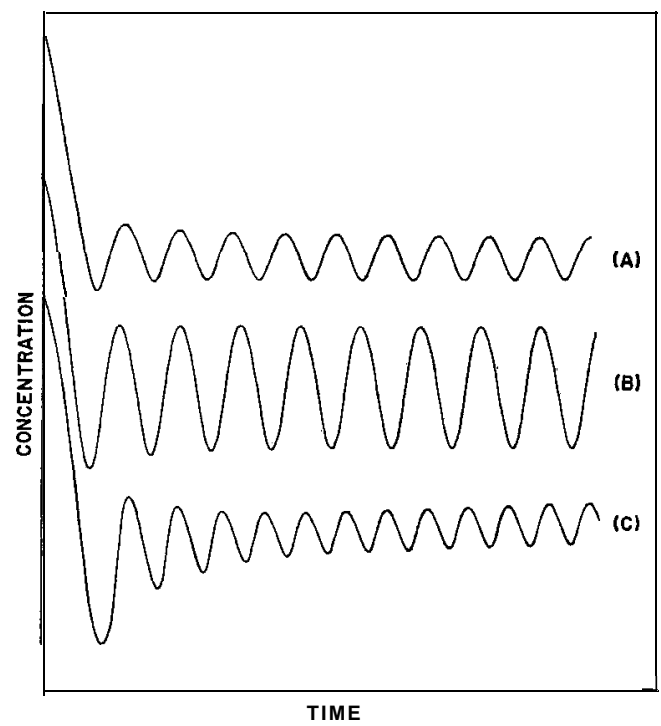

Fig. 11. Sustained oscillation. Parameters listed in Table 1 were used.

Table 1. Relation between $k_{1} / k_{4}$ value and sustained oscillation.

\begin{tabular}{ccccc}
\hline$k_{1}$ & $k_{4}$ & $k_{1} / k_{\mathbf{4}}$ Value & Initial $X_{4}$ & Fig. 11 \\
\hline & & & & \\
0.800 & 8.20 & 0.08 & 0.543 & (A) \\
0.453 & 6.33 & 0.07 & 0.543 & \\
0.050 & 0.72 & 0.07 & 0.543 & (C) \\
0.025 & 0.36 & 0.07 & 0.543 & \\
\hline
\end{tabular}


value. In order to determine the allowable range for $k_{1} / k_{4}$ ratio, the computations were performed with changing the $k_{4}$ value under the fixed value of $k_{1}$. The results are listed in Table 2. It is clear that the only very narrow range of the ratio, 0.07-0. 09, is essential for the generation of the sustained oscillation.

Table 2. Effect of $k_{1} / k_{4}$ value on oscillation behavior.

\begin{tabular}{ccccc}
\hline$k_{1}$ & $k_{4}$ & $k_{1} / k_{4}$ Value & Initial $\mathrm{X}_{4}$ & \\
\hline 0.453 & 1.16 & 0.39 & 0.543 & Damped \\
0.453 & 4.21 & 0.11 & 0.543 & Damped \\
0.453 & 5.45 & 0.09 & 0.543 & Sustained \\
0.453 & 7.00 & 0.07 & 0.543 & Sustained \\
0.453 & 8.00 & 0.06 & 0.543 & Divergent
\end{tabular}

\section{Effect of initial concentration of $\boldsymbol{X}_{4}$ on oscillation}

The combinations of $k_{1}$ and $k_{4}$ are divided into two classes under fixing the $k_{1} / k_{4}$ ratio at 0.07 .

(A) $k_{4}$ value is below 1.0 (example: $k_{1} / k_{4}=0.025 / 0.36$ ).

(B) $k_{4}$ value is above 1.0 (example ; $k_{1} / k_{4}=0.435 / 6.33$ ).

In each case, the oscillatory behavior of the system was computed with changing the initial concentration of $X_{4}$. This situation is corresponding to that the external perturbation was introduced to the system so as to increase or decrease the value (concentration) of $X_{4}$. In the case of (A), the oscillatory behavior with changing the initial concentration of $X_{4}$ from 0.0 to 1.0 is shown in Table 3. The initial value of $X_{4}$ above 0.5 has broken the sustained oscillation of $X_{4}$. The average value of $X_{4}$ at the stationary state was changing downward with increase in the initial value of $X_{4}$. In the case of (B), the results are listed in Table 4. The average value of $X_{4}$ at the stationary state was changing upward in proportion to the increase in the initial value of $X_{4}$.

For both cases, (A) and (B), the ratio of $k_{1} / k_{4}$ was the same, although the real value of $k_{1}$ and $k_{4}$ were different from each other. The unified rate constant at the summing point, $\mathbf{Z}=k_{1} /\left(k_{4} X_{4}\right)$ was the same for both the cases, when it was compared under the same initial value of $X_{4}$. Furthermore, as can be

Table 3. Influence of the initial concentration of $X_{4}$ on the oscillation behavior (A) $\left(k_{1}=0.025, k_{4}=0.36\right)$.

\begin{tabular}{|c|c|c|c|c|}
\hline Initial $X_{4}$ & $\begin{array}{l}\text { Average } X_{4} \text { at } \\
\text { stationary state }\end{array}$ & Pattern of $X_{4}$ & $\begin{array}{c}\text { Pattern } \\
\text { corresponds }\end{array}$ & $s$ to \\
\hline 0.0 & 0.075 & Sustained & Fig. & 4 \\
\hline$\downarrow$ & 0.050 & Sustained & Fig. & 4 \\
\hline $\begin{array}{l}\downarrow \\
0.6 \\
\downarrow \\
0.7 \\
\downarrow\end{array}$ & $\begin{array}{c}0.020 \downarrow \\
\downarrow \\
0.010 \\
\downarrow\end{array}$ & $\begin{array}{c}\text { Damped } \downarrow \\
\downarrow \\
\text { Damped } \\
\downarrow\end{array}$ & $\begin{array}{l}\text { Fig. } \\
\text { Fig. }\end{array}$ & 2 \\
\hline
\end{tabular}


Table 4. Influence of the initial concentration of $X_{4}$ on oscillation behavior (B) $\left(k_{1}=0.453, k_{4}=6.33\right)$.

\begin{tabular}{|c|c|c|c|}
\hline Initial $X_{4}$ & $\begin{array}{l}\text { Average } X_{4} \text { at } \\
\text { stationary state }\end{array}$ & Pattern of $\boldsymbol{X}_{4}$ & $\begin{array}{c}\text { Pattern } \\
\text { corresponds to }\end{array}$ \\
\hline 0.0 & 0.040 & Damped & Fig. 2 \\
\hline $\begin{array}{l}\downarrow \\
0.4\end{array}$ & 0.075 & Sustained & Fig. \\
\hline 0.5 & $0.090 \downarrow$ & Sustdined & Fig. \\
\hline 0.8 & 0.125 & Damped & Fig. \\
\hline $\begin{array}{c}\downarrow \\
\downarrow\end{array}$ & $\begin{array}{c}0.135 \\
\downarrow\end{array}$ & Damped & Fig. \\
\hline
\end{tabular}

seen in the last equation in the simultaneous differential equation (2), $X_{4}$ was consumed by $Y_{2}$ but not by the feedback loop. These considerations suggest us that the response of the system against the initial value (external perturbation) would be the same for both cases, in spite of the differences in the $k_{1}$ and $k_{4}$ values. The real computations indicated quite different patterns on the stationary values of $X_{4}$ as shown already, contrary to the expectation. In the case of (A), $X_{4}$ value at the stationary state decreased with the increase in the initial value, whereas in the case of (B), just the reverse was observed. The reason, why such the different responses were derived under the same value of $Z$, can not be clarified at the present time.

\section{Effect of $\boldsymbol{k}_{2}$ value on oscillation}

The effect of $k_{2}$ value in the step of $X_{2} \rightarrow X_{3}$ on the oscillatory behavior of the system was estimated by the simulation under the conditions that $k_{1} / k_{4}$ $(=0.07)$ and the initial value of $X_{4}(=0.543)$ were fixed. As listed in Table 5, the increase in the $k_{2}$ value accompanied the change in the $X_{4}$ pattern in order of divergent, damped, sustained, damped. The sustained oscillation on $X_{4}$ was observable in a narrow range of $k_{2}$ value, 0.916-2.0. In order to estimate the effect of the combination of $k_{2}$ value with the initial value of $X_{4}$, the computations were performed with changing the initial value of $X_{4}$ under selected $k_{2}$

Table 5. Effect of $k_{2}$ value on oscillation behavior.

\begin{tabular}{cc}
\hline$k_{2}$ & Pattern of $X_{4}$ \\
\hline$\downarrow$ & 1 \\
0.90 & Divergent \\
$\downarrow$ & Damped \\
1.50 & Sustained \\
$\downarrow$ & $\downarrow$ \\
2.00 & Sustained \\
$\downarrow$ & $\downarrow$ \\
3.00 & Damped \\
$\downarrow$ & $\downarrow$ \\
3.47 & Damped \\
$\downarrow$ & $\downarrow$ \\
\hline
\end{tabular}


values, 0.916, 2.00 and 3.00. The results are listed in Table 6. With $k_{2}=0.916$, the pattern of $X_{4}$ changed continuously from divergent to damped oscillation and the stationary value of $\boldsymbol{X}_{4}$ changed downward with increase in the initial value of $X_{4}$.

Table 6. Relation between $k_{2}$ value and the initial concentration of $X_{4}$.

\begin{tabular}{|c|c|c|}
\hline$k_{2}$ & $0.0 \longrightarrow$ Initial $X_{4}$ & $\begin{array}{l}\text { Change in average } X_{i} \\
\text { at stationary state }\end{array}$ \\
\hline 0.916 & Div. $\rightarrow$ Sus. $\rightarrow$ Sus. $\rightarrow$ Dam. $\rightarrow$ Dam. & Downward \\
\hline 2.00 & Sưs. $\rightarrow$ sus. $\rightarrow$ sus. $\rightarrow$ sus. $\rightarrow$ sus. & Scarcely \\
\hline 3.00 & Dam. $\rightarrow$ Dam.+ Dam. $\longrightarrow$ Dam. $\rightarrow$ Dam. & Scarcely \\
\hline
\end{tabular}

Div., Divergent ; Dam., Damped oscillation ; Sus., Sustained oscillation.

It is noteworthy that the oscillation profiles were quite stable for $k_{2}=2.00$ and 3.00. The profiles were not altered by the increase in the initial value of $X_{4}$. Furthermorr, the stationary values of $X_{4}$ in the case of $k_{2}=2.00$ and 3.00 were shifted very scarcely with increase in the initial value of $X_{4}$. Thus, the feedback control system is able to maintain the concentration of a certain intermediate at constant value against the external pcrturbation.

\section{Effect of $\boldsymbol{k}_{3}$ value on oscillation}

The effect of $k_{3}$ value which is the rate constant for the step of $\boldsymbol{X}_{3} \rightarrow \boldsymbol{X}_{4}$ on the oscillatory behavior of the system was estimated under fixing the value of $k_{1} / k_{4}=\mathbf{0 . 0 7}$ and the initial value of $X_{4}=0.543$. The computed results are summarized in Table 7 . Similar to the effect of $k_{2}$, the increase in the $k_{3}$ value accompanied the alteration of pattern of $X_{4}$. The effect of the initial concentration of $X_{4}$ on the pattern was examined with fixing the $k_{3}$ value at $0.700,0.746,2.83$ and 3.50 , respectively. In the cases of $k_{3}=2.83$ and 3.50 , the patterns did not change with increase in the initial value of $X_{4}$ and the average $X_{4}$ value at the stationary state was also remained unchanged as shown in Table 8.

There seems to be no clear evidences conflicting to the argument that the feedback system is a prime mode to control the metabolism or the enzymatic chain reactions in vivo. However, it does not necessarily mean that any type

Table 7. Effect of $k_{3}$ value on oscillation behavior

\begin{tabular}{cc}
\hline$k_{3}$ & Pattern of $X_{4}$ \\
\hline$\downarrow$ & Divergent \\
$\downarrow$ & Damped \\
0.700 & Sustained \\
$\downarrow$ & $\downarrow$ \\
$\downarrow .746$ & Sustained \\
2.83 & $\downarrow$ \\
$\downarrow$ & Damped \\
3.50 & $\downarrow$ \\
$\downarrow$ & \\
\hline
\end{tabular}


Table 8. Relation between $k_{3}$ value and the initial concentration of $X_{4}$.

\begin{tabular}{|c|c|c|}
\hline$k_{3}$ & $0.0 \stackrel{\text { Initial } X_{4}}{\longrightarrow} 0.5 \stackrel{\text { }}{\longrightarrow}$ & $\begin{array}{l}\text { Change in average } X_{4} \\
\text { at stationary state }\end{array}$ \\
\hline 0.700 & Sus. $\rightarrow$ Sus. $\rightarrow$ Dam. $\longrightarrow$ Dam. & Downward \\
\hline 0.746 & slls. $\longrightarrow$ Sus. $\longrightarrow$ Dam. $\rightarrow$ Dam. & Downward \\
\hline 2.83 & Sus. $\longrightarrow$ sus. $\longrightarrow$ Sus. & No \\
\hline 3.50 & Dam. $\longrightarrow$ Dam. $\longrightarrow$ Dam. & No \\
\hline
\end{tabular}

of feedback system is able to realize the capability of autocontrolling the enzymatic chain reactions. Some limited types of feedback system may act as a controlling device in organisms. The dispensable conditions imposed on the feedback system as controlling device have not yet been established. In other words, the behaviors of biochemical feedback system have not been surveyed enough to establish their qualification for the autocontrolling device.

In the present study, a model of biochemical feedback system was assumed and it was analyzed by analog computer simulation to follow especially the oscillatory behavior and capability to treat the external perturbation. The obtained results may be summarized as follows: (1) For realizing the sustained oscillation, the strictly limited parameter-set was needed. The sustained oscillations of the metabolic intermediate in vivo have been widely observed. The results of the present computation, therefore, suggest that the in vivo feedback system itself would be regulated further by systems of higher order. It is interesting that oscillating properties of feedback system were considered to be, in the electronic or mechanical dynamics, undesired one, while the biochemical systems generate intentionally the oscillation of metabolic intermediates. (2) It is obvious that the feedback system assumed here exhibits the several ways by which the external perturbation are treated. As described already, under the suitable parameter-set, the average value of $X_{4}$ at the stationary state was held at a constant value regardless of the magnitude of the perturbation which was introduced in the form of $X_{4}$ to the system, and the perturbation was stored on the intermediate other than $X_{4}$ at the stationary state. Thus, in the feedback system, the perturbation can be treated at positions other than the original position where the perturbation was introduced. This will be specially meaningful when one argues the effect of the external perturbation on the metabolic pathway in vivo. (3) Another peculiar results are shown in Tables 3 and 4. In the case of (A), the increase in the magnitude of the external perturbation caused the decrease in the average value of $X_{4}$ at the stationary state, whereas in the case of (B), the reverse was observed. Thus, the effects of perturbation on the average stationary value of intermediate are changing delicately according to the variation of parameter-set. This may infer that one's simple and qualitative inference on the effect of the perturbation on the biochemical feedback system should be avoided.

In the present model for a chemical feedback system, two important assumptions were made: First, at the summing point, the operation represented by 
equation (2) was assumed, and second, the $X_{4}$ was assumed to be lost only by the constant $Y_{2}$. On the basis of molecular mechanism, the above operation at the summing point would be realized by some type of allosteric enzymes, and the second assumption would be accomplished by a followed feedback system which can control the flow or consumption of $X_{4}$ at $Y_{2}$ value.

\section{ACKNOWLEDGEMENT}

The authors wish to thank Prof. Matsui and Dr. Eguchi of the Biotron Institute of Kyushu University for their encouragement throughout the present study. This research was supported by a grant of scientific research from the Ministry of Education.

\section{REFERENCES}

Goodwin, C. 1965 Oscillatory behavior in enzymatic control processes. Adv. Enzyme Regulation, 3: $425-4: 38$

Higgins, J., R. Frankel, E. Hulme, A. Lucas and G. Rangazas 1973 The control theoretic approach to the analysis of glycolytic oscillators. In "Biological and Biochemical Oscillators," Academic Press, New York

Morales, M. and D. McKay 1967 Biochemical oscillation in controlled systems. Biophys. J., 7: $621-625$

Pavlidis, T. 1973 Biological Oscillators: Their Mathematical Analysis. Academic Press, New York

Sompayrac, L. and 0. Maalфe 1973 Autorepressor model for control of DNA replication. Nature New Biology, 241: 133-135 